\title{
Isolation, Characterization, and Application of Nanocellulose from Oil Palm Empty Fruit Bunch Fiber as Nanocomposites
}

\author{
N. S. Lani, ${ }^{1}$ N. Ngadi, ${ }^{1}$ A. Johari, ${ }^{2}$ and M. Jusoh ${ }^{1}$ \\ ${ }^{1}$ Department of Chemical Engineering, Universiti Teknologi Malaysia, 81310 Skudai, Johor, Malaysia \\ ${ }^{2}$ Institute of Hydrogen Economy, Faculty of Chemical Engineering, Universiti Teknologi Malaysia, 81310 Johor Bahru, Johor, Malaysia \\ Correspondence should be addressed to N. Ngadi; norzita@cheme.utm.my
}

Received 21 April 2014; Revised 3 June 2014; Accepted 3 June 2014; Published 8 July 2014

Academic Editor: Prashant Kumar

Copyright (c) 2014 N. S. Lani et al. This is an open access article distributed under the Creative Commons Attribution License, which permits unrestricted use, distribution, and reproduction in any medium, provided the original work is properly cited.

\begin{abstract}
Nanocomposites, consisting of a polymeric matrix and nanosized elements as reinforcement, have attracted significant scientific attention because of their high mechanical performance. A large variety of nanocomposites have been prepared using bio-based materials as a matrix and nanoreinforcement, so that it can reduce the dependence on nondegradable products and move to a sustainable materials basis. The objective of this study was to isolate nanocellulose from empty fruit bunch (EFB) fiber and their reinforcing effect on polyvinyl alcohol (PVA)/starch blend films. A series of PVA/starch films with different content of nanocellulose were prepared by solution casting method. Nanocellulose fiber with diameters ranging from 4 to $15 \mathrm{~nm}$ has been successfully prepared. On the other hand, PVA/starch films reinforced with nanocellulose fiber possess significantly improved properties compared to unreinforced film. From the results, PVA/starch films with the addition of $5 \%(\mathrm{v} / \mathrm{v})$ of nanocellulose exhibited best combination of properties. This nanocomposite was found to have tensile strength at about $5.694 \mathrm{MPa}$ and elongation at break was $481.85 \%$. In addition to good mechanical properties, this nanocomposite has good water resistance and biodegradability.
\end{abstract}

\section{Introduction}

Nowadays, the demands of plastics materials are increasing rapidly, especially in food packaging application. Packaging is used to keep the product from surrounding and to maintain the food product quality. The levels of chemical migration from the packaging have to be lower than control safe level [1], or otherwise the food might be contaminated and consequently risks the consumer safety. Moreover, another issue and consideration is the severe problems created by food packages to the environment since most of these products are not normally environmentally friendly and nonbiodegradable.

The use of polyvinyl alcohol (PVA) particularly in commercial industry has arisen worldwide. This is due to their unique chemical and physical properties, as well as being nontoxic, highly crystalline, and water-soluble polymer and having good film-forming and high hydrophilic properties. Furthermore, PVA is highly recognized as a biodegradable polymer. However, the main disadvantage of PVA is their price which is quite expensive. Therefore, PVA can be blended with other natural polymers in order to reduce the materials costs and thus make PVA more economical to use.

Starch is one of the most widely used in blends with PVA as PVA/starch blend would increase biodegradability of PVA. It has been scientifically proven through extensive worldwide studies by many researchers [2-4] and by Ishigaki et al. [5]. Tang and Alavi [6] mentioned that both starch and polyvinyl alcohol are polar substances that have hydroxyl groups in their chemical structure, which are able to form intermolecular and intramolecular hydrogen bond. However, the major disadvantages of these PVA/starch blends are particularly poor water barrier properties, generally attributed to the very large number of hydroxyl groups along with their intrinsic hydrophilicity. Therefore, several studies proposed the incorporation of fillers in a nanoscale size into PVA/starch blends in order to improve their water barrier properties.

Most of the previous studies have investigated the use of nanofillers such as nanosilicon dioxide [7, 8], montmorillonite clay $[9,10]$, sodium montmorillonite clay [11], and nanoparticles of poly(methyl methacrylate-co-acrylamide) 
[12]. However, these nanoparticles had no significant influence on biodegradability of films. For that reason, this research was conducted for the purpose of improvement in the properties of PVA and starch blend by using nanocellulose from EFB fiber as reinforcement. Furthermore, very limited studies have been reported concerning the nanocellulose reinforced PVA/starch blend films compared to other fillers.

Cellulose extracted from empty fruit bunch (EFB) fiber has attracted growing interest because of their unique characteristics, including low cost, lower density, high specific strength, good thermal properties, and biodegradable. Oil palm EFB fiber is a natural fiber which has great relevance to Malaysia, as a large quantity of the biomass is generated by oil palm industries. The total amount of EFB produced was estimated to be more than 10 million tons per year, whereby only $10 \%$ of the EFB is used and the rest are abundant [13].

Natural fibers are mainly composed of cellulose, hemicellulose, and lignin. Generally, the EFB fiber contains about 40-50\% cellulose, $20-30 \%$ hemicellulose, and $20-30 \%$ lignin with moisture content of about $10-15 \%$, which has highest composition of cellulose compared to coir, corn, bagasse, and kenaf fiber. Furthermore, a comprehensive research and review article dealing with isolation of cellulose fiber in nanoparticle size by sulfuric acid hydrolysis was published by many researchers [14-22].

Besides food packaging applications, nanocomposites based on polymer matrix have a great potential for a broad range of application fields such as automotive, aerospace, optoelectronics, and biomedical industry. Owing to their nanoscale size features and high aspect ratio, they not only possess excellent mechanical properties comparable to composite reinforced with microsized fillers, but they also display outstanding combination of optical, electrical, thermal, magnetic, and other physic-chemical properties.

The aims of this study were to isolate nanocellulose from EFB fiber via acid hydrolysis process. The properties of untreated and nanocellulose fiber were investigated using Fourier transform infrared (FTIR) spectroscopy, X-ray diffraction (XRD), and thermogravimetric (TG) analysis. The morphology of the isolated nanofibers was also characterized using transmission electron microscopy (TEM) analysis. The effect of nanocellulose content, isolated from EFB fiber, on the properties of PVA/starch films was also explored.

\section{Experimental}

2.1. Materials and Chemicals. Empty fruit bunch fibers were collected from Johor, Malaysia, and were used as raw material in this study. Sodium chlorite, acetic acid, sodium sulfite, sodium hydroxide, sulfuric acid, polyvinyl alcohol, starch, and glycerol meanwhile were purchased from Sigma-Aldrich. All the chemicals are reagent grade and were used as received.

2.2. Isolation of Nanocellulose. A $0.7 \%(\mathrm{w} / \mathrm{v})$ sodium chlorite $\left(\mathrm{NaClO}_{2}\right)$ solution was prepared and then acetic acid was added to acidify the $\mathrm{NaClO}_{2}$ solution until the $\mathrm{pH}$ reached 4. The fibers were boiled in $\mathrm{NaClO}_{2}$ solution for 2 hours at $70-80^{\circ} \mathrm{C}$ whereby the ratio of fiber to $\mathrm{NaClO}_{2}$ solution was set to $1: 50$. The bleaching process was repeated for four or five times until fiber became white and then filtered. After being filtered, the residue was washed for several times with distilled water and dried in air. The bleached cellulose obtained was heated to about 70 to $80^{\circ} \mathrm{C}$ in $5 \%$ (w/v) sodium sulfite solution for 2 hours. The fibers were filtered, washed, and dried in air. After being dried, the fibers were treated in $17.5 \%(\mathrm{w} / \mathrm{v})$ sodium hydroxide $(\mathrm{NaOH})$ solution for 2 hours. The residue was washed for several times with distilled water and dried in air.

After alkali treatment of fibers, the cellulose materials were hydrolyzed in $64 \mathrm{wt} \%$ sulfuric acid solution under strong agitation at $45^{\circ} \mathrm{C}$ for 45 minutes. Then, cold water was added to stop the reaction. The diluted suspension was then centrifuged at $11000 \mathrm{rpm}$ for 10 minutes to obtain precipitate. This process was repeated until the $\mathrm{pH}$ of the suspension reach 5 then dialysis was carried out for 3 days.

2.3. Preparation of Nanocomposite. An aqueous solution containing PVA, starch, glycerol, and nanocellulose fiber were used to cast the film. The PVA and starch solution (70:30 wt\%) were prepared by dissolving PVA powder in $100 \mathrm{~mL}$ distilled water at $97^{\circ} \mathrm{C}$ for 30 minutes, while being stirred with a mechanical stirrer until PVA particles were completely dissolved in water. After the PVA had fully dissolved, starch was then added and the mixture is again heated and stirred at $97^{\circ} \mathrm{C}$ for 30 minutes [23]. Glycerol was added to the mixed solution at a $30 \mathrm{wt} \%$ ratio based on total weight of starch and PVA [24]. Five sets of nanocomposites with a nanocellulose content of $5,10,15$, and $20 \%(\mathrm{v} / \mathrm{v})$ with respect to the volume of PVA and starch solution were prepared. A solution of $5 \mathrm{~g}$ mixed polymer was cast on Petridish plate and allow it to dry at room temperature for 2 days. After the film is completely dry, the film was peeled off from the plate.

2.4. Fourier Transform Infrared Spectroscopy. FTIR spectra of raw EFB and nanocellulose fibers were obtained by using Spectrum One-Perkin Elmer with software spectrum v5.02. The fibers were crushed into small particles and then blended with potassium bromide $(\mathrm{KBr})$ followed by pressing the mixture into ultrathin pellets. In this analysis, ratio of fiber to $\mathrm{KBr}$ was approximately 1:99. A total of 100 scans were recorded in the range $370-4000 \mathrm{~cm}^{-1}$ with a resolution of $2 \mathrm{~cm}^{-1}$ for each sample.

2.5. X-Ray Diffraction. The XRD patterns for fibers and composite films were measured using Ni-filter $\mathrm{Cu} K \alpha$ radiation, with a wavelength of $0.1541 \mathrm{~nm}$ at $40 \mathrm{kV}$ and $40 \mathrm{~mA}$. Powder samples were scanned in $2 \theta$ range varying from $10^{\circ}$ to $50^{\circ}$. Equation (1) was used to calculate the crystallinity index of the fibers:

$$
\text { Crystallinity index }=\frac{I_{200}-I_{\mathrm{am}}}{I_{200}} \times 100 \%,
$$

where $I_{200}$ is the height of the 200 peak, which represents both crystalline and amorphous material while $I_{\mathrm{am}}$ is the lowest 
height between the 200 and 110 peaks, which represents amorphous material.

2.6. Scanning Electron Microscopy. The surface morphology of the film samples was investigated by using a JEOL JSM6390LV scanning electron microscope, with the accelerating voltage of $50 \mathrm{kV}$. All specimens were coated with gold and then observed.

2.7. Transmission Electron Microscopy. TEM images were obtained using a JEM-2100 transmission electron microscope operated at $120 \mathrm{kV}$. For TEM sample preparation, the nanocellulose particles were deposited on a carbon-coated grid by placing a drop of a very dilute cellulose nanofiber suspension on the grid and then allowed to dry in order to evaporate the liquid.

2.8. Mechanical Properties. The tensile strength and elongation at break of the composite films were measured on the $2.5 \mathrm{kN}$ Lloyd tensile tester according to the ASTM D882. Samples with rectangular shape were cut into $60 \mathrm{~mm} \times 13 \mathrm{~mm}$ strips from the films. The cut films were conditioned at the temperature and relative humidity of $23 \pm 2^{\circ} \mathrm{C}$ and $50 \pm 10 \%$, respectively, for more than 40 hours prior to measurement of mechanical properties. Tensile testing was conducted at a cross-head speed of $10 \mathrm{~mm} /$ minute and a gauge length of $40 \mathrm{~mm}$. Six samples were tested for each set and averaged.

2.9. Water Absorption Properties. The water absorption capacity of the composite films was measured by drying the film in a vacuum oven at $50^{\circ} \mathrm{C}$ for 24 hours. Then, the film was cooled in desiccator and immediately weighted. The conditioned film was fully immersed in distilled water for 5 minutes. Subsequently, the remaining water on the surface of film was removed by using filter paper and immediately weighted. All measurements were performed for four specimens and averaged. The water absorption capacity was calculated using the following:

$$
\text { Water absorption capacity }=\frac{W_{A}-W_{I}}{W_{I}} \times 100 \% \text {, }
$$

where $W_{A}$ is the weight of film after being immersed in distilled water and $W_{I}$ is the initial dry weight of the film.

2.10. Soil Burial Degradation Properties. The films were cut into $50 \mathrm{~mm} \times 50 \mathrm{~mm}$ strips and then were weighted. The degradation test was carried out by burying the films in the soil placed in a flower pot at the depth of $5 \mathrm{~cm}$. The humidity, temperature, and moisture values were negligible and do not require any adjustment in order to provide a realistic environment, where soil humidity, temperature, and type and amount of microorganisms are in less control and change with weather. At a 15-day interval, the films were taken out carefully from the soil. The remaining soil on the surface of the film was removed and then weighted. The degradation rate in the soil burial test was determined by calculating the weight loss of the films with time using the following:

$$
\text { Degradation rate }=\frac{W_{I}-W_{H}}{W_{I}} \times 100 \%,
$$

where $W_{H}$ is the dry weight of the specimen after being buried in the soil and $W_{I}$ is the initial dry weight of the specimen.

\section{Results and Discussion}

3.1. Fourier Transform Infrared Spectroscopy. The FTIR spectra spectroscopy is an indispensable technique for tracking changes in the chemical structures of the fibers after the various treatments which they received. The FTIR spectra of the raw EFB fiber and nanocellulose fibers are shown in Figure 1.

As shown in Figure 1, untreated and treated fibers presented two main regions of absorbance; the first one at low wavelengths $\left(500-1750 \mathrm{~cm}^{-1}\right)$ and the second one at higher wavelengths $\left(2800-3500 \mathrm{~cm}^{-1}\right)$. This observation is in agreement with the study done by Morán et al. [15]. The absorption peaks located in the ranges $3300-3450 \mathrm{~cm}^{-1}$ and $2890-2900 \mathrm{~cm}^{-1}$ in both samples are attributed to the hydroxyl group and aliphatic saturated $\mathrm{C}-\mathrm{H}$ stretching vibration, respectively $[15,25,26]$. The peaks appearing from 1330 to $1360 \mathrm{~cm}^{-1}$ are due to the bending vibration of the $\mathrm{C}-\mathrm{H}$ and $\mathrm{C}-\mathrm{O}$ groups in the polysaccharide aromatic rings in cellulose, hemicelluloses, and lignin [27]. Meanwhile, stretching of C$\mathrm{O}$ and $\mathrm{O}-\mathrm{H}$ was reflected by peaks appearing in the region $1050-1070 \mathrm{~cm}^{-1}$ [28] and the peak at $1055 \mathrm{~cm}^{-1}$ is due to the bending vibration of the $\mathrm{C}-\mathrm{O}-\mathrm{C}$ pyranose ring [14].

Stretching vibrations of $\mathrm{C}=\mathrm{C}$ bonds in the lignin are found in the $1608-1623 \mathrm{~cm}^{-1}$, whereby the peak at $1623 \mathrm{~cm}^{-1}$ is caused by vibrations of $\mathrm{C}=\mathrm{C}$ bonds in the benzene stretching ring. Meanwhile, the vibration of the aromatic rings can be seen at $1608 \mathrm{~cm}^{-1}$ in the spectra of the untreated fiber, which is attributed to the $\mathrm{C}=\mathrm{C}$ stretching of the carboxyl groups [28]. In addition, lignin presented characteristic peak at $1432 \mathrm{~cm}^{-1}$ was due to the presence of methoxy-O- $\mathrm{CH}_{3}$, while the vibration peak at $1259 \mathrm{~cm}^{-1}$ has been related to the $\mathrm{C}-\mathrm{O}-\mathrm{C}$ stretching of the aryl-alkyl ether [29]. Disappearance of all these peaks from the spectra of the treated fibers can be ascribed to treatment of the EFB fibers with sodium hydroxide followed by acid hydrolysis as these treatments are known to remove most of the lignin from the EFB fibers.

Furthermore, the most noticeable difference between spectra of the untreated fiber and those of the chemically treated ones is disappearance of the vibration peak observed at $1736 \mathrm{~cm}^{-1}$ from the spectra of the alkali treated fibers. This peak is also absent in the sulfuric acid hydrolysed cellulose. The peak observed at $1736 \mathrm{~cm}^{-1}$ in the spectra of the untreated fibers is likely to be associated with the $\mathrm{C}=\mathrm{O}$ stretching in the acetyl and uronic ester groups of hemicelluloses or the ester carbonyl groups in the $p$-coumaric units of the lignin. Another possibility is that carboxyl or aldehyde absorption could be arising from the opened terminal glucopyranose rings or oxidation of the $\mathrm{C}-\mathrm{OH}$ groups $[30,31]$. This peak 


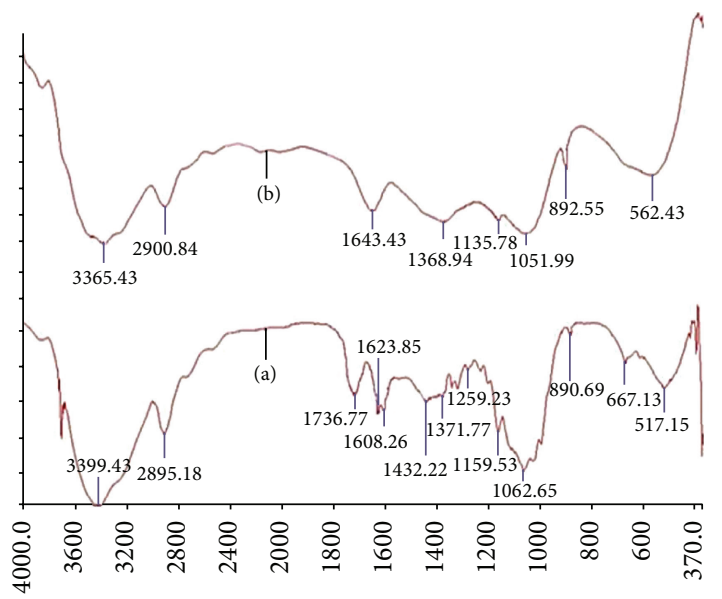

FIGURE 1: FTIR spectra of (a) untreated fiber and (b) nanocellulose fiber.

has disappeared from the spectra of cellulose fibers and it is believed to be due to removal of hemicellulose by alkali treatment.

By comparing the spectra of the untreated and treated EFB fibers, the peak presents at $1646 \mathrm{~cm}^{-1}$ corresponding to the treated fibers is related to the absorption of water by cellulose. Appearance of this peak in the spectra of the cellulose fibers is probably due to reaction of sodium hydroxide with the hydroxyl groups of celluloses and subsequent formation of water molecules [27]. As pointed out by Abraham et al. [32], even though the treated fibers were subjected to the drying process, the water adsorbed in the cellulose molecules is very difficult to be extracted due to the cellulose-water interaction.

From the FTIR analysis, it has been concluded that almost all the lignin and hemicelluloses were removed after chemical treatment. This arises from the absence of the absorption bands in-between 1500 and $1600 \mathrm{~cm}^{-1}$ and 1730 and $1740 \mathrm{~cm}^{-1}$, which relates to the lignin and hemicelluloses components, respectively. Therefore, it can be stated that the nanocellulose obtained in this study was almost pure, which contains a very small amounts of lignin and other noncellulosic substances.

3.2. X-Ray Diffraction. The X-ray diffraction patterns of the untreated and treated EFB fiber are shown in Figure 2 meanwhile the diffraction patterns of PVA/starch film and $\mathrm{PVA} /$ starch reinforced with nanocellulose composite film are shown in Figure 3.

As can be seen in Figure 2, both fibers had diffraction peaks at around $2 \theta=22^{\circ}$ and $2 \theta=14^{\circ}$, representing the presence of crystalline and amorphous cellulose structure, respectively. The crystallinity index of untreated EFB fiber (calculated by Segal formula) was approximately 38\% while that of nanocellulose fiber was estimated $73 \%$. By comparing the results, nanocellulose from EFB fiber obtained in this study exhibited higher crystallinity than those produced by Fahma et al. [33] and Jonoobi et al. [31], which is around 59 and 69\%, respectively. According to Jonoobi et al. [31],

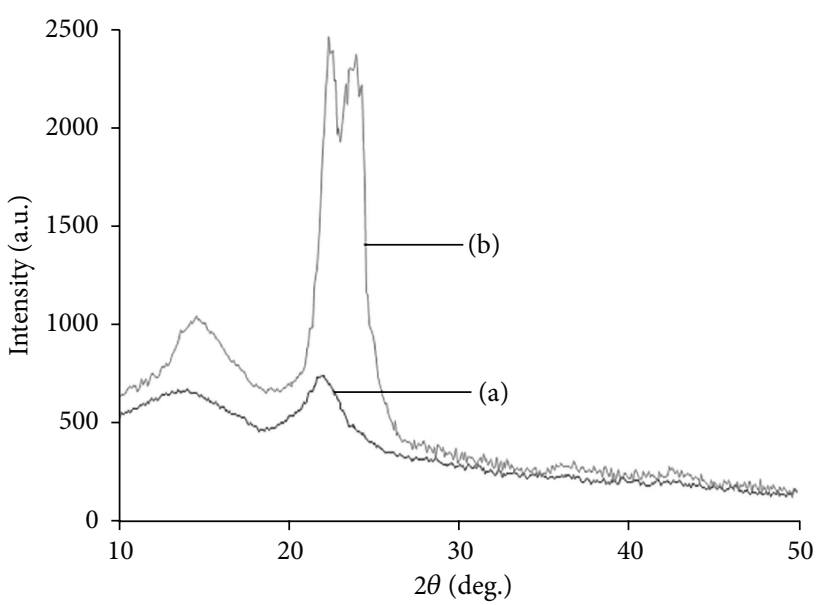

FIGURE 2: X-ray diffraction patterns for (a) untreated fiber and (b) nanocellulose fiber.

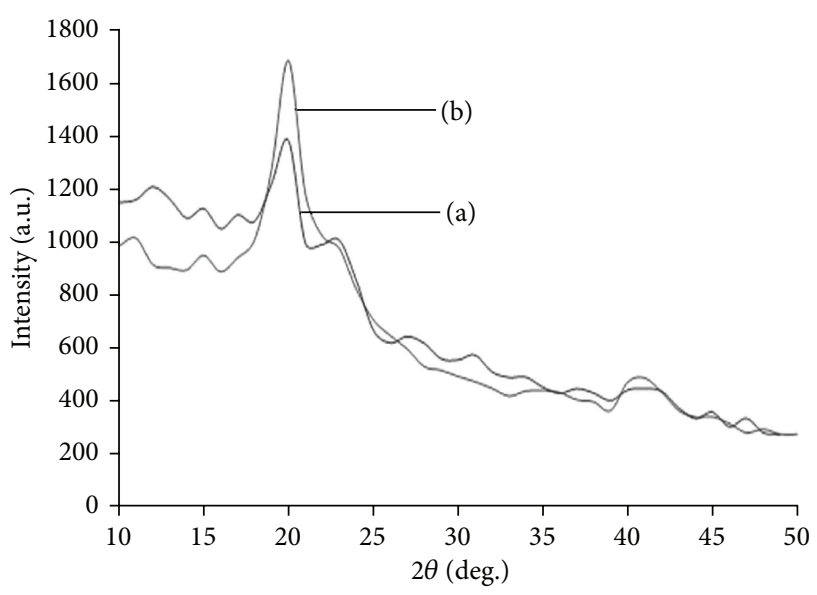

FIGURE 3: X-ray diffraction patterns for (a) PVA/starch blend film and (b) PVA/starch reinforced with 5\% (v/v) nanocellulose composite film.

the higher crystallinity of nanofiber was due to the peeling reactions that take place in the amorphous areas during the hydrolysis process. Because of the ability of sulfuric acid to penetrate into the amorphous region, the glycosidic bonds were cleaved under hydrolytic conditions and finally releasing individual crystallites $[34,35]$.

Generally, cellulose in natural fibers comes in four types of cellulose that are classified as types I, II, III, and IV. It is interesting to note that the untreated EFB fiber shows typical cellulose I structure, based on the fact that there is no doublet in the main peak at $2 \theta=22^{\circ}[36,37]$. After treating the EFB fiber with sodium hydroxide and sulfuric acid, the crystalline peak appears as a doublet at $2 \theta=22^{\circ}$ and $2 \theta=23^{\circ}$, representing transformation of native cellulose from cellulose I to cellulose II. A significant variation in the diffraction pattern of untreated and cellulose fibers can be explained by the replacement of $\mathrm{OH}$ groups by $\mathrm{ONa}$ groups during alkaline treatment. Subsequent rinsing with water will remove the linked $\mathrm{Na}$-ions and thus causing the cellulose to 
be converted to a new crystalline structure, which is cellulose II [38].

Meanwhile, based on the XRD diffractogram shown in Figure 3, the crystallinity indexes of PVA/starch blend films and PVA/starch reinforced with nanocellulose composite film were found to be 30.77 and $50.38 \%$, respectively. It has been shown that the presence of nanocellulose fibers in the blend film essentially increased the crystallinity of the composite film. The reason of the increment of crystallinity was due to the nucleation effects, whereby the nanocelluloses were acted as an agent of nucleation for the crystal growth [39]. The crystalline regions of the composites behave as barriers and any change in crystallinity may affect the final properties of the composite. Since PVA/starch nanocomposite film has a higher degree of crystallinity than blend film, a higher water resistance would be expected, as water absorption occurs almost entirely in the amorphous region of a polymeric system [40].

3.3. Transmission Electron Microscopy. Figure 4 shows the TEM micrograph of a dilute suspension of nanocellulose from EFB fiber prepared by acid hydrolysis.

The analysis of the TEM micrograph revealed that the aqueous suspension of nanocellulose fiber consist of rodlike nanoparticles, whereby there were some nanoparticles agglomerated in the forms of bundles while some of them were well separated. According to Othman et al. [41], agglomeration is generally due to the Van der Waals attraction forces between nanoparticles. Moreover, it can be seen that the diameter of the nanocellulose fiber was found to be in the range of $4-15 \mathrm{~nm}$. Interestingly, the diameter of the nanocellulose obtained in this study was smaller than those produced by an acid hydrolysis from different sources such as cotton linter $(14.6 \pm 3.9 \mathrm{~nm})$ by Roohani et al. [42], microcrystalline cellulose $(28 \pm 9 \mathrm{~nm})$ by Bilbao-Sainz et al. [43], and sisal fiber ( $30.9 \pm 12.5 \mathrm{~nm})$ by Morán et al. [15] as well as those produced by mechanical treatment originating from rutabaga fiber [36], bamboo fiber [44], and rubberwood [31], for which the range of diameter has been reported as 5$20 \mathrm{~nm}, 30-80 \mathrm{~nm}$, and $10-90 \mathrm{~nm}$, respectively.

3.4. Scanning Electron Microscopy. The surface morphology of the composite film was also studied by SEM analysis. Figure 5 shows the electron micrograph for the surface of PVA/starch composite film with $5 \%$ and $20 \%$ (v/v) nanocellulose content. It can be seen that the nanocellulose fibers at low content were better dispersed in the PVA/starch matrix, as there is no rough surface or large agglomerates (Figure 5(a)). Meanwhile, agglomeration of nanocellulose was observed at PVA/starch composite film with $20 \%(\mathrm{v} / \mathrm{v})$ nanocellulose content, which contributes to poor final properties of composite films.

3.5. Mechanical Properties. Figure 6 indicates the effect of nanocellulose content on tensile strength and elongation at break of the nanocomposite films.

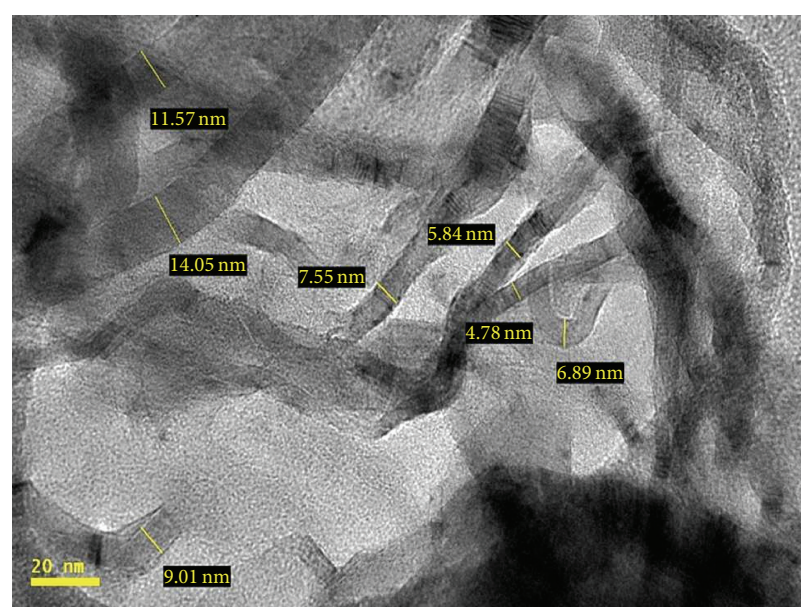

FIGURE 4: TEM image of nanocellulose from EFB fiber.

From Figure 6, it is observed that the tensile strength of the PVA/starch blend film was $3.830 \mathrm{MPa}$. When nanocellulose fiber was added into the PVA and starch solution, the tensile strength was increased along with an increase in nanocellulose content, and the highest tensile strength occurred at $10 \%(\mathrm{v} / \mathrm{v})$, which was around $7.094 \mathrm{MPa}$. When the nanocellulose content exceeded $10 \%(\mathrm{v} / \mathrm{v})$, the tensile strength of the blend film decreased along with an increase in nanocellulose content, but it was still much higher than that of the unreinforced film.

The same trend from addition of nanocellulose fiber content can also be found for the elongation at break. It was found that the elongation at break had the highest value when $5 \%(\mathrm{v} / \mathrm{v})$ nanocellulose was used during the nanocomposite preparation, whereby the elongation at break of the nanocomposite film increased by $30.54 \%$ in comparison to the unreinforced film. An addition of $10 \%(\mathrm{v} / \mathrm{v})$ nanocellulose content however had deterioration effect for elongation at break as a sharp decrease was observed from $481.85 \%$ to $312.18 \%$. Then, the elongation at break of the nanocomposite film decreased slowly from $312.18 \%$ to $288 \%$ along with an increase in nanocellulose content from $10 \%$ to $20 \%(\mathrm{v} / \mathrm{v})$.

The enhancement in tensile strength and elongation at break at low content of fillers was due to very fine nature of nanocellulose fiber and well dispersion of nanocellulose through the PVA/starch matrix. Meanwhile, according to Das et al. [24], the remarkable increase in the mechanical properties indicated the presence of intermolecular interactions between nanocellulose and PVA or nanocellulose and starch, which disturbs the interchain force of attraction of the PVA/starch. On the other hand, the reason of the reduction of tensile strength and elongation at break was the fact that the excess amount of nanocellulose fiber leads to an increase in intermolecular interaction that might compete with interactions between PVA, starch, and nanocellulose fiber. Therefore, the miscibility and compatibility of PVA, starch, and nanocellulose were reduced, which decreased the tensile strength and elongation at break of nanocomposite films. 


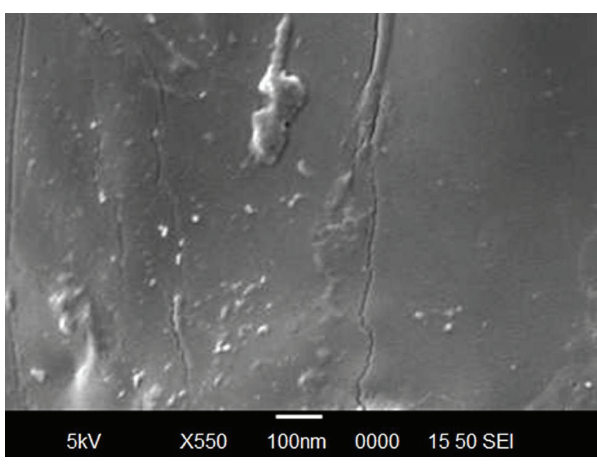

(a)

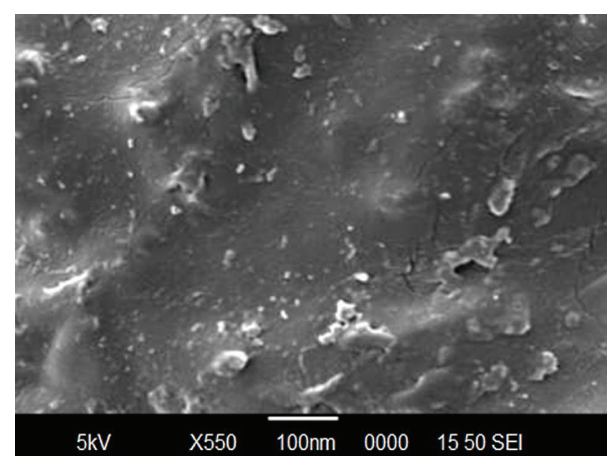

(b)

FIGURE 5: SEM image of PVA/starch composite film with (a) 5\% (v/v) and (b) 20\% (v/v) nanocellulose content.

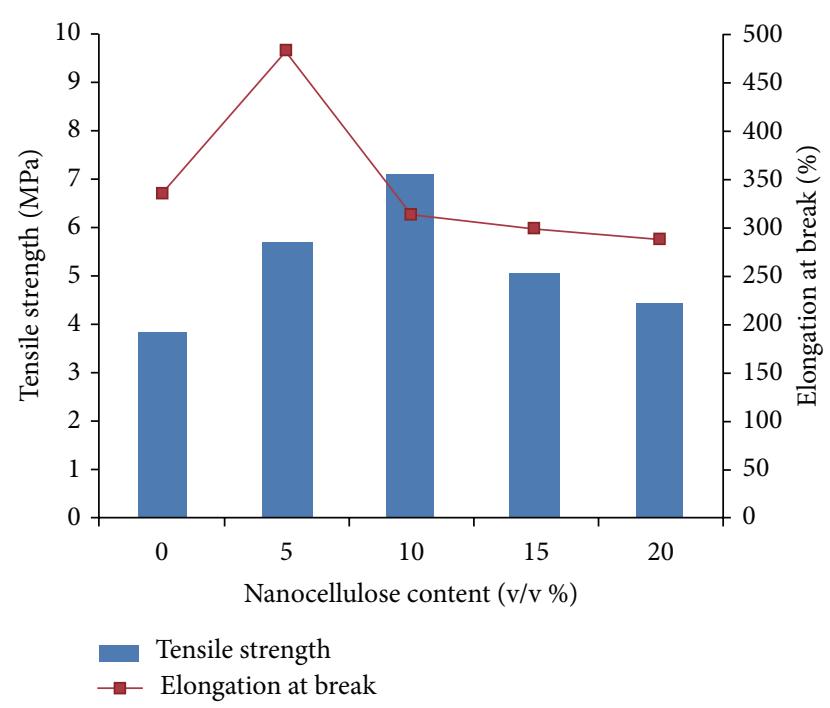

FIGURE 6: The dependence of the tensile strength and elongation at break on the nanocellulose content.

3.6. Water Absorption Properties. Figure 7 shows that the water absorption capacity of PVA/starch/nanocellulose composite films depended on the amount of nanocellulose loading.

Comparing the water absorption capacity of PVA/starch blend film and nanocellulose fiber reinforced PVA/starch composite, it is noted that the percentage of water absorption of all nanocellulose fiber reinforced films was lower, indicating that films that contain nanocellulose have a good preventive ability against water. This result illustrates that the water absorption properties were improved by the presence of nanocellulose fiber in the blend films. The possible reason for this kind of behavior was related to the formation of the network structure between the nanocellulose particles and PVA/starch components, which prevented the water molecules from dissolving and improved the water resistance of the film. The presence of the nanocellulose fibers means that the pathway for water molecules to diffuse into the composites is altered from the direct diffusion to a tortuous path, which leads to the improvement in water barrier

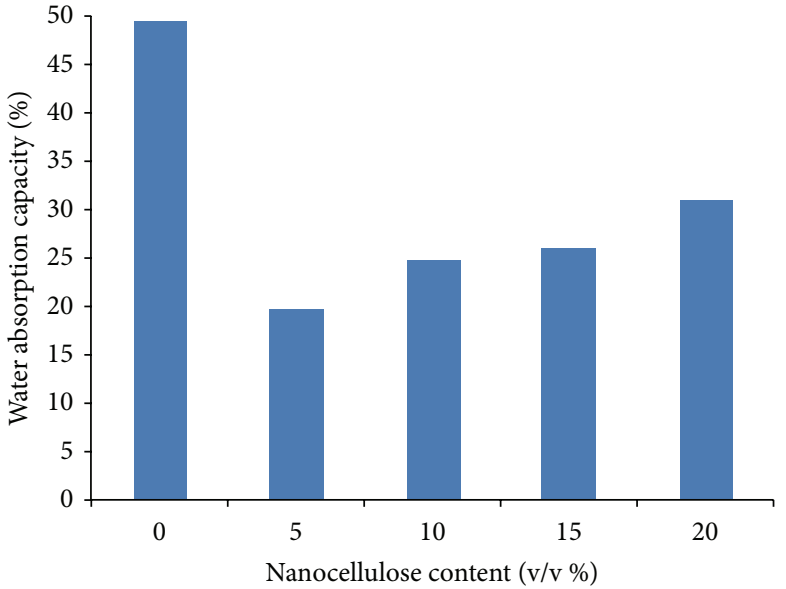

FIgURE 7: Percentage of water absorption capacity for PVA/starch reinforced with nanocellulose fiber at different amount.

properties. Meanwhile, Alhuthali et al. [45] in their study stated that the high aspect ratio of nanocellulose may have also contributed to a reduction in absorbed water.

Some differences are observed by increasing nanocellulose content in PVA/starch blend films during water absorption tests. By comparison, an increment of water absorption capacity was observed with increasing nanocellulose content, which is possibly due to the poor dispersion of the nanocellulose fiber (Figure 5). It can be observed that the water absorption of PVA/starch/nanocellulose films increased from 19.71 to $31.06 \%$ with cellulose content from 5\% to $20 \%$ $(\mathrm{v} / \mathrm{v})$. It can be explained by the formation of some large accumulated particles, whereby the large accumulation of nanocellulose particles can generate voids which can absorb water molecules and therefore increase the percentage of water absorption [46].

3.7. Soil Burial Degradation Properties. Degradability of composites is important when a polymeric system is applied in daily lives as its weight loss degree has a direct influence on the environment. In this study, the effect of different amount 


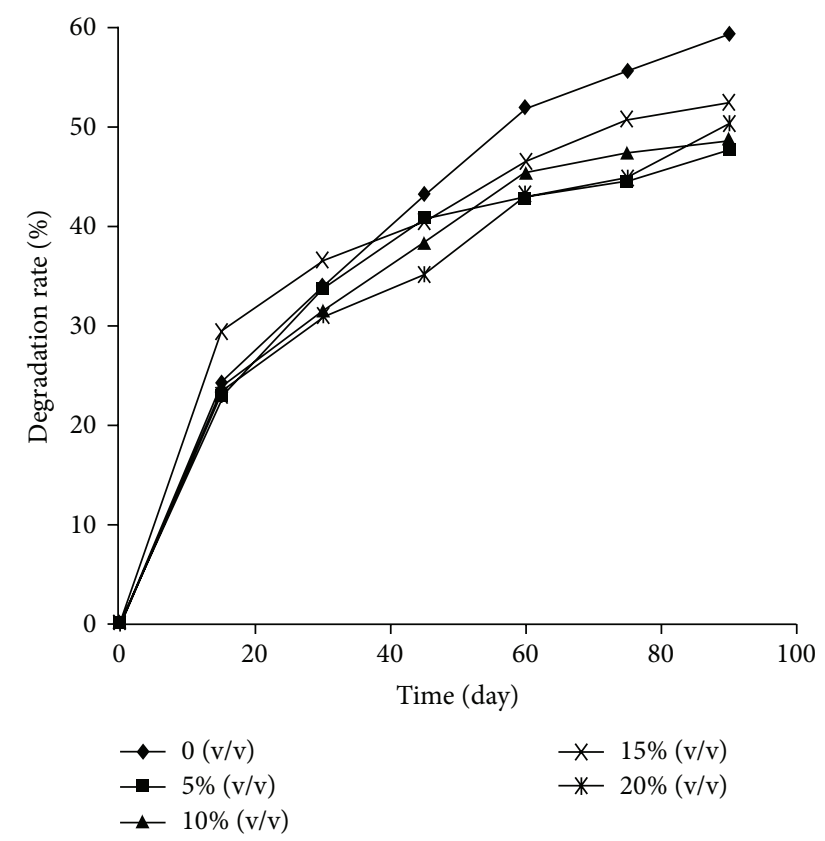

FIGURE 8: Degradability of PVA/starch nanocomposite films in the soil burial test.

of nanocellulose fiber on film weight loss rate was conducted via soil burial test and the results were presented in Figure 8.

Results showed that the degradation rate of PVA/starch blend film was higher than PVA/starch films that contained nanocellulose fiber, whereby the PVA/starch blend films without the addition of nanocellulose were able to lose its weight up to $59.39 \%$ after being buried for 90 days. It is believed that the faster disintegration of PVA/starch blend films can be explained by the ability of composite film to absorb higher amount of water. This is because the water absorbed by the films caused the microorganisms such as algae, bacteria, and fungi to attach and grow, since the films have become the main energy sources of the microbial growth. This result is consistent with the result from water absorption test of PVA/starch nanocomposite films reflected in Figure 8 and thus illustrates that the addition of nanocellulose fiber prevented water to be diffused into the films and the growth of microorganism occurred slowly. Furthermore, the study also found that the degradation rate of PVA/starch nanocomposite after being buried for 90 days is slightly lower than that of the PVA/starch blend film.

\section{Conclusion}

In this study, cellulose and nanocellulose were successfully extracted from palm oil EFB fiber. It has been proved that alkali treatment seems to be effective in the removal of lignin and hemicelluloses components. In addition, the chemical treatment used in this study was able to achieve improvement in the properties of cellulose and nanocellulose, compared to that observed in previous studies. Evidence of noncellulosic components loss can be observed in the FTIR spectra, whereby there is no main absorption band of lignin and hemicelluloses at $1500-1600 \mathrm{~cm}^{-1}$ and $1730-1740 \mathrm{~cm}^{-1}$ after treating with $\mathrm{NaOH}$. On the other hand, the crystallinity index of the fiber increases after each treatment. Higher crystallinity value of the fiber is expected to provide better reinforcement and therefore better properties of the composite films. TEM observation revealed that acid hydrolysis was able to produce nanocellulose from EFB fiber with a diameter in the range of $4-15 \mathrm{~nm}$.

Successful improvements of nanocellulose reinforced $\mathrm{PVA} / \mathrm{starch}$ film can be seen in terms of mechanical and water absorption properties due to the good compatibility between the nanocellulose and the polymer matrix. Furthermore, the properties of nanocomposite films were also affected by different nanocellulose content. The reinforcement effect at low nanocellulose content in the PVA/starch matrix is much stronger because the excess nanocellulose tended to agglomerate. The PVA/starch reinforced with nanocellulose fiber had a tensile strength, elongation at break, and water absorption capacity of approximately $5.694 \mathrm{MPa}, 481.85 \%$, and $19.71 \%$ at an addition of $5 \%(\mathrm{v} / \mathrm{v})$ nanocellulose content.

\section{Conflict of Interests}

The authors declare that there is no conflict of interests regarding the publication of this paper.

\section{References}

[1] K. A. Barnes, R. Sinclair, and D. Watson, Chemical Migration and Food Contact Materials, Elsevier B.V., Woodhead, UK, 1st edition, 2007.

[2] M. A. L. Russo, R. Truss, and P. J. Halley, "The enzymatic hydrolysis of starch-based PVOH and polyol plasticised blends," Carbohydrate Polymers, vol. 77, no. 3, pp. 442-448, 2009.

[3] N. Tudorachi, C. N. Cascaval, M. Rusu, and M. Pruteanu, “Testing of polyvinyl alcohol and starch mixtures as biodegradable polymeric materials," Polymer Testing, vol. 19, no. 7, pp. 785-799, 2000.

[4] Y. Yun, Y. J. Wee, H. S. Byun, and S. D. Yoon, "Biodegradability of chemically modified starch (RS4)/PVA blend films: part 2," Journal of Polymers and the Environment, vol. 16, no. 1, pp. 12$18,2008$.

[5] T. Ishigaki, Y. Kawagoshi, M. Ike, and M. Fujita, "Biodegradation of a polyvinyl alcohol-starch blend plastic film," Journal of Microbiology and Biotechnology, vol. 15, no. 3, pp. 321-327, 1999.

[6] X. Tang and S. Alavi, "Recent advances in starch, polyvinyl alcohol based polymer blends, nanocomposites and their biodegradability," Carbohydrate Polymers, vol. 85, no. 1, pp. 716, 2011.

[7] S. Tang, P. Zou, H. Xiong, and H. Tang, "Effect of nano- $\mathrm{SiO}_{2}$ on the performance of starch/polyvinyl alcohol blend films," Carbohydrate Polymers, vol. 72, no. 3, pp. 521-526, 2008.

[8] Z. Abbasi, "Water resistance, weight loss and enzymatic degradation of blends starch/polyvinyl alcohol containing $\mathrm{SiO}_{2}$ nanoparticle," Journal of the Taiwan Institute of Chemical Engineers, vol. 43, no. 2, pp. 264-268, 2012.

[9] K. Majdzadeh-Ardakani and B. Nazari, "Improving the mechanical properties of thermoplastic starch/poly(vinyl alcohol)/clay nanocomposites," Composites Science and Technology, vol. 70, no. 10, pp. 1557-1563, 2010. 
[10] I. Spiridon, M. C. Popescu, R. Bodârlǎu, and C. Vasile, "Enzymatic degradation of some nanocomposites of poly(vinyl alcohol) with starch," Polymer Degradation and Stability, vol. 93, no. 10, pp. 1884-1890, 2008.

[11] M. T. Taghizadeh, Z. Abbasi, and Z. Nasrollahzade, "Study of enzymatic degradation and water absorption of nanocomposites starch/polyvinyl alcohol and sodium montmorillonite clay," Journal of the Taiwan Institute of Chemical Engineers, vol. 43, no. 1, pp. 120-124, 2012.

[12] S. D. Yoon, M. H. Park, and H. S. Byun, "Mechanical and water barrier properties of starch/PVA composite films by adding nano-sized poly(methyl methacrylate-co-acrylamide) particles," Carbohydrate Polymers, vol. 87, no. 1, pp. 676-686, 2012.

[13] A. B. A. Hariharan and H. P. S. Abdul-Khalil, "Lignocellulosebased hybrid bilayer laminate composite: part I-studies on tensile and impact behavior of oil palm fiber-glass fiberreinforced epoxy resin," Journal of Composite Materials, vol. 39, no. 8, pp. 663-684, 2005.

[14] A. Mandal and D. Chakrabarty, "Isolation of nanocellulose from waste sugarcane bagasse (SCB) and its characterization," Carbohydrate Polymers, vol. 86, no. 3, pp. 1291-1299, 2011.

[15] J. I. Morán, V. A. Alvarez, V. P. Cyras, and A. Vázquez, "Extraction of cellulose and preparation of nanocellulose from sisal fibers," Cellulose, vol. 15, no. 1, pp. 149-159, 2008.

[16] J. Araki, M. Wada, S. Kuga, and T. Okano, "Flow properties of microcrystalline cellulose suspension prepared by acid treatment of native cellulose," Colloids and Surfaces A: Physiochemical and Engineering Aspects, vol. 142, no. 1, pp. 75-82, 1998.

[17] M. J. Cho and B. D. Park, "Tensile and thermal properties of nanocellulose-reinforced poly(vinyl alcohol) nanocomposites," Journal of Industrial and Engineering Chemistry, vol. 17, no. 1, pp. 36-40, 2011.

[18] F. Fahma, S. Iwamoto, N. Hori, T. Iwata, and A. Takemura, "Effect of pre-acid-hydrolysis treatment on morphology and properties of cellulose nanowhiskers from coconut husk," Cellulose, vol. 18, no. 2, pp. 443-450, 2011.

[19] L. N. Ludueña, A. Vecchio, P. M. Stefani, and V. A. Alvarez, "Extraction of cellulose nanowhiskers from natural fibers and agricultural byproducts," Fibers and Polymers, vol. 14, no. 7, pp. 1118-1127, 2013.

[20] J. P. S. Morais, M. F. Rosa, M. M. S. De Souza Filho, L. D. Nascimento, D. M. Do Nascimento, and A. R. Cassales, "Extraction and characterization of nanocellulose structures from raw cotton linter," Carbohydrate Polymers, vol. 91, no. 1, pp. 229-235, 2013.

[21] J. F. Revol, H. Bradford, J. Giasson, R. H. Marchessault, and D. G. Gray, "Helicoidal self-ordering of cellulose microfibrils in aqueous suspension," International Journal of Biological Macromolecules, vol. 14, no. 3, pp. 170-172, 1992.

[22] S. M. L. Rosa, N. Rehman, M. I. G. De Miranda, S. M. B. Nachtigall, and C. I. D. Bica, "Chlorine-free extraction of cellulose form rice husk and whisker isolation," Carbohydrate Polymers, vol. 87, no. 2, pp. 1131-1138, 2012.

[23] W. A. W. A. Rahman, L. T. Sin, A. R. Rahmat, and A. A. Samad, "Thermal behaviour and interactions of cassava starch filled with glycerol plasticized polyvinyl alcohol blends," Carbohydrate Polymers, vol. 81, no. 4, pp. 805-810, 2010.

[24] K. Das, D. Ray, N. R. Bandyopadhyay, S. Sahoo, A. K. Mohanty, and M. Misra, "Physico-mechanical properties of the jute micro/nanofibril reinforced starch/polyvinyl alcohol biocomposite films," Composites Part B: Engineering, vol. 42, no. 3, pp. 376-381, 2011.

[25] B. M. Cherian, A. L. Leão, S. F. de Souza et al., "Cellulose nanocomposites with nanofibres isolated from pineapple leaf fibers for medical applications," Carbohydrate Polymers, vol. 86, no. 4, pp. 1790-1798, 2011.

[26] H. P. S. A. Khalil, H. Ismail, H. D. Rozman, and M. N. Ahmad, "Effect of acetylation on interfacial shear strength between plant fibres and various matrices," European Polymer Journal, vol. 37, no. 5, pp. 1037-1045, 2001.

[27] M. Le Troedec, D. Sedan, C. Peyratout et al., "Influence of various chemical treatments on the composition and structure of hemp fibres," Composites A: Applied Science and Manufacturing, vol. 39, no. 3, pp. 514-522, 2008.

[28] M. K. Nacos, P. Katapodis, C. Pappas et al., "Kenaf xylan-a source of biologically active acidic oligosaccharides," Carbohydrate Polymers, vol. 66, no. 1, pp. 126-134, 2006.

[29] H. Yang, R. Yan, H. Chen, D. H. Lee, and C. Zheng, "Characteristics of hemicellulose, cellulose and lignin pyrolysis," Fuel, vol. 86, no. 12-13, pp. 1781-1788, 2007.

[30] A. Alemdar and M. Sain, "Isolation and characterization of nanofibers from agricultural residues-wheat straw and soy hulls," Bioresource Technology, vol. 99, no. 6, pp. 1664-1671, 2008.

[31] M. Jonoobi, A. Khazaeian, P. M. Tahir, S. S. Azry, and K. Oksman, "Characteristics of cellulose nanofibers isolated from rubberwood and empty fruit bunches of oil palm using chemomechanical process," Cellulose, vol. 18, no. 4, pp. 1085-1095, 2011.

[32] E. Abraham, B. Deepa, L. A. Pothan et al., "Extraction of nanocellulose fibrils from lignocellulosic fibres: a novel approach," Carbohydrate Polymers, vol. 86, no. 4, pp. 1468-1475, 2011.

[33] F. Fahma, S. Iwamoto, N. Hori, T. Iwata, and A. Takemura, "Isolation, preparation, and characterization of nanofibers from oil palm empty-fruit-bunch (OPEFB)," Cellulose, vol. 17, no. 5, pp. 977-985, 2010.

[34] A. Dufresne, Nanocellulose: From Nature to High Performance Tailored Materials, Walter de Gruyter, Berlin, Germany, 1st edition, 2012.

[35] R. Li, J. Fei, Y. Cai, Y. Li, J. Feng, and J. Yao, "Cellulose whiskers extracted from mulberry: a novel biomass production," Carbohydrate Polymers, vol. 76, no. 1, pp. 94-99, 2009.

[36] A. Bhatnagar and M. Sain, "Processing of cellulose nanofiberreinforced composites," Journal of Reinforced Plastics and Composites, vol. 24, no. 12, pp. 1259-1268, 2005.

[37] D. Klemm, B. Heublein, H. P. Fink, and A. Bohn, "Cellulose: fascinating biopolymer and sustainable raw material," Angewandte Chemie, vol. 44, no. 22, pp. 3358-3393, 2005.

[38] M. J. John and R. D. Anandjiwala, "Recent developments in chemical modification and characterization of natural fiberreinforced composites," Polymer Composites, vol. 29, no. 2, pp. 187-207, 2008.

[39] B. Alexandre, D. Langevin, P. Médéric et al., "Water barrier properties of polyamide 12/montmorillonite nanocomposite membranes: structure and volume fraction effects," Journal of Membrane Science, vol. 328, no. 1-2, pp. 186-204, 2009.

[40] M. Mucha, S. Ludwiczak, and M. Kawinska, "Kinetics of water sorption by chitosan and its blends with poly(vinyl alcohol)," Carbohydrate Polymers, vol. 62, no. 1, pp. 42-49, 2005.

[41] S. H. Othman, S. Abdul Rashid, T. I. Mohd Ghazi, and N. Abdullah, "Dispersion and stabilization of photocatalytic $\mathrm{TiO}_{2}$ 
nanoparticles in aqueous suspension for coatings applications," Journal of Nanomaterials, vol. 2012, Article ID 718214, 10 pages, 2012.

[42] M. Roohani, Y. Habibi, N. M. Belgacem, G. Ebrahim, A. N. Karimi, and A. Dufresne, "Cellulose whiskers reinforced polyvinyl alcohol copolymers nanocomposites," European Polymer Journal, vol. 44, no. 8, pp. 2489-2498, 2008.

[43] C. Bilbao-Sainz, J. Bras, T. Williams, T. Sénechal, and W. Orts, "HPMC reinforced with different cellulose nano-particles," Carbohydrate Polymers, vol. 86, no. 4, pp. 1549-1557, 2011.

[44] W. Chen, H. Yu, and Y. Liu, "Preparation of millimeter-long cellulose I nanofibers with diameters of $30-80 \mathrm{~nm}$ from bamboo fibers," Carbohydrate Polymers, vol. 86, no. 2, pp. 453-461, 2011.

[45] A. Alhuthali, I. M. Low, and C. Dong, "Characterisation of the water absorption, mechanical and thermal properties of recycled cellulose fibre reinforced vinyl-ester eco-nanocomposites," Composites Part B: Engineering, vol. 43, no. 7, pp. 2772-2781, 2012.

[46] W. Zou, J. Peng, Y. Yang, L. Zhang, B. Liao, and F. Xiao, "Effect of nano- $\mathrm{SiO}_{2}$ on the performance of poly(MMA/BA/MAA)/EP," Materials Letters, vol. 61, no. 3, pp. 725-729, 2007. 

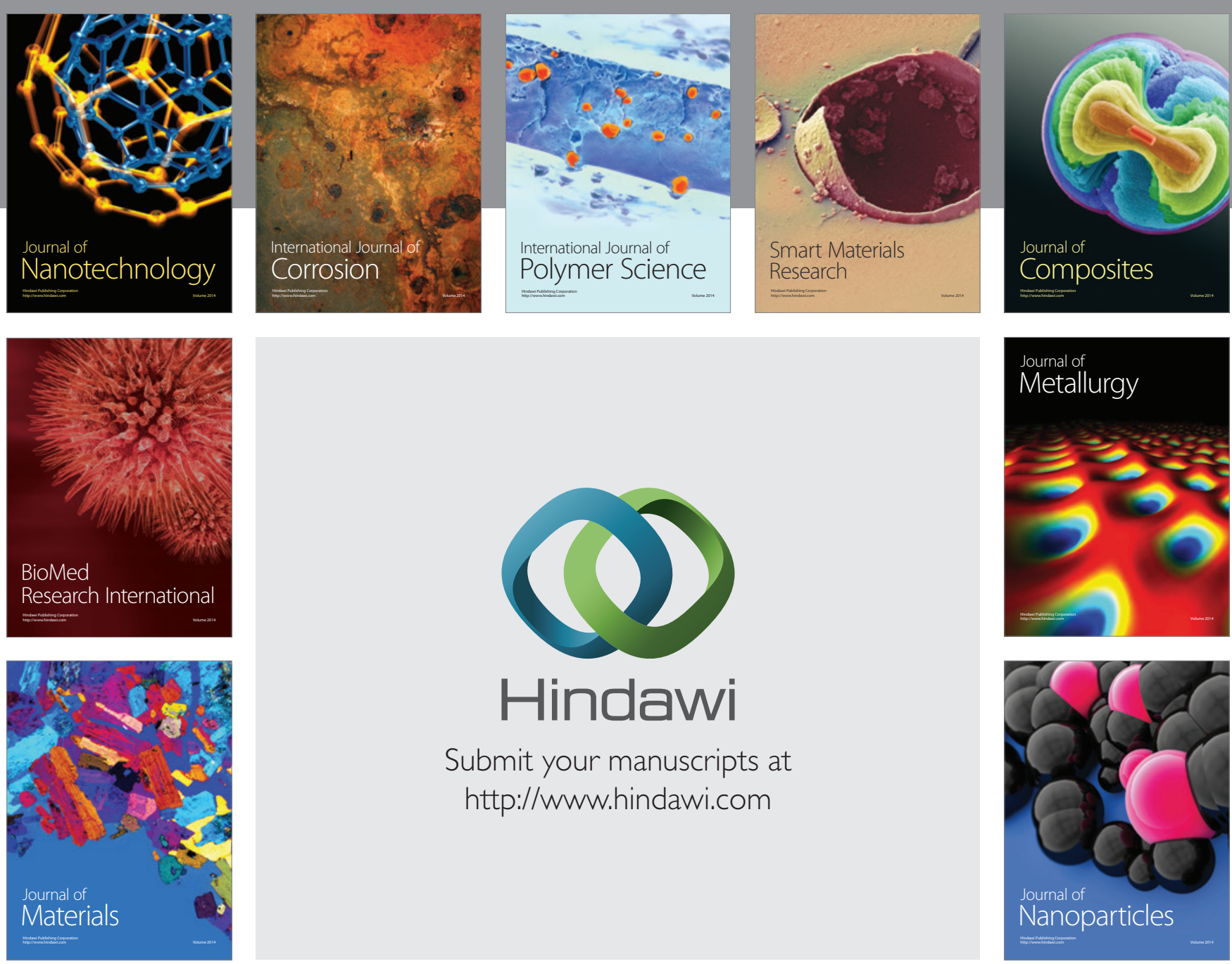

Submit your manuscripts at http://www.hindawi.com
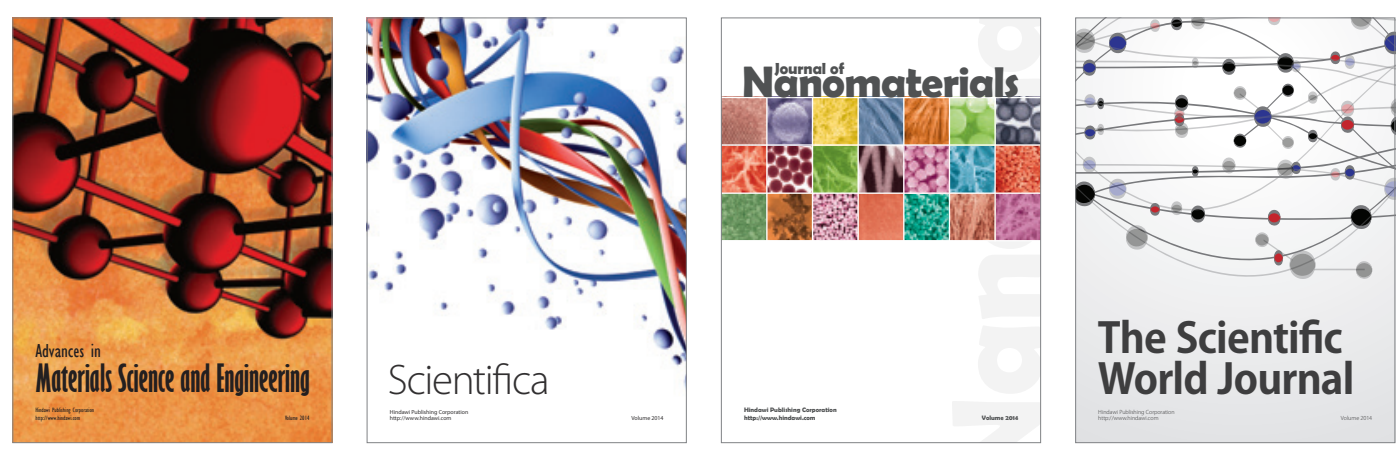

\section{The Scientific World Journal}
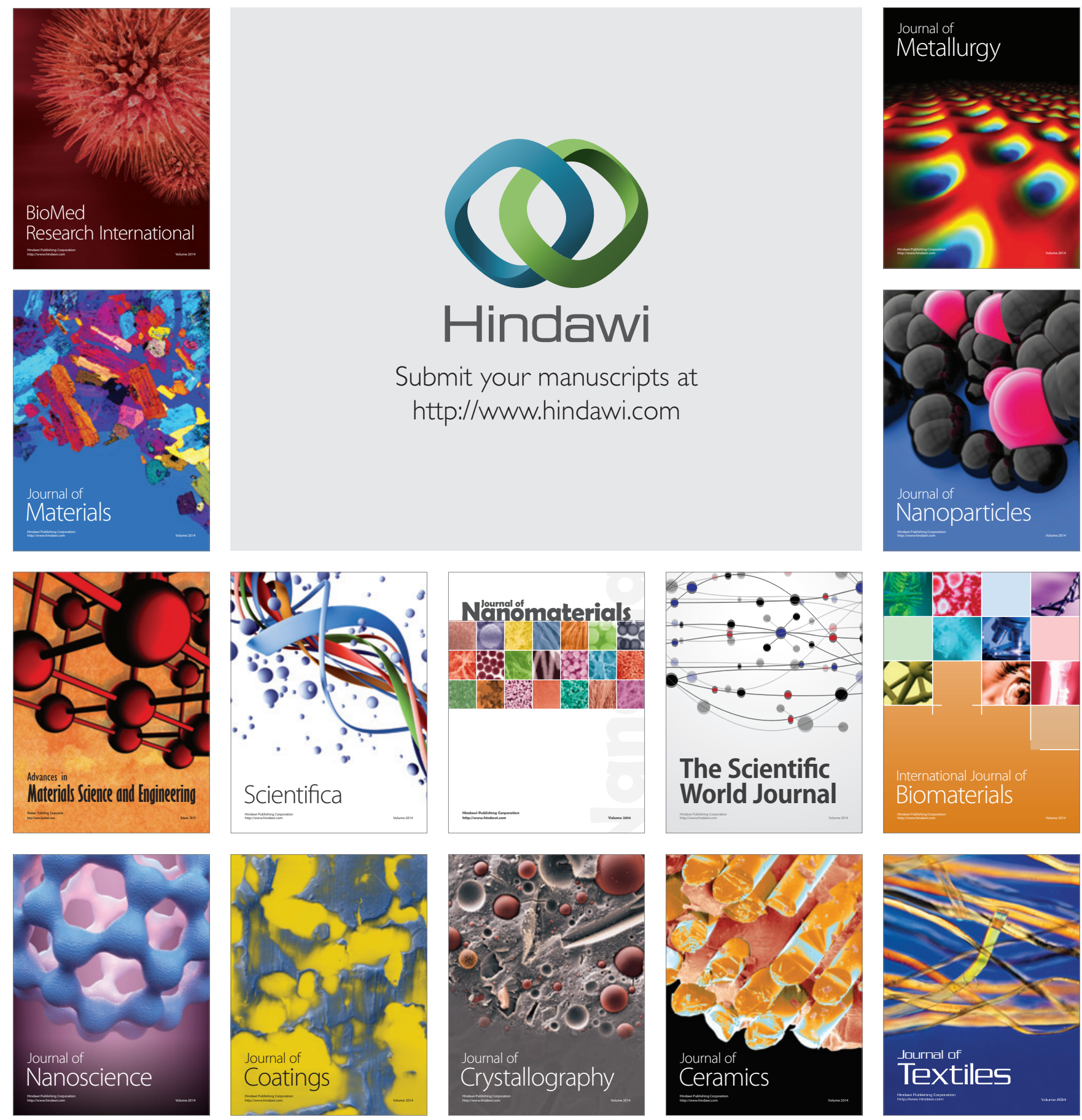